\title{
Soft tissue sarcomas of the extremities
}

\author{
BlAKE A. MORRISON, MD
}

$\mathbf{S}$

oft tissue sarcomas are a diverse group of neoplasms that arise in the connective tissues throughout the body. They account for approximately $1 \%$ of adult malignancies and $7 \%$ to $15 \%$ of pediatric malignancies. About $50 \%$ to $60 \%$ of sarcomas occur in the extremities, and although they are rare, they are responsible for more deaths than testicular cancer, Hodgkin's disease, and thyroid cancer combined (1). These tumors are notorious for recurring and metastasizing - often with devastating results—despite apparently complete resection.

\section{INCIDENCE}

The National Cancer Institute's Cancer Surveillance, Epidemiology, and End-Result (SEER) Program in 1996 reported 6400 new cases of soft tissue sarcoma, including 3500 in males and 2900 in females (2), for a male-to-female ratio of about 1.2:1. Deaths were reported in 1800 males and 1900 females, so although males are more likely to develop sarcoma, females are more likely to die of it.

The 1996 SEER data revealed significant changes from 1990: 700 more new cases and 600 more deaths were reported (3). Devesa studied the decades from the 1970s through the 1990s, however, and observed no such increase (4). This discrepancy may be attributed to the large number of Kaposi's sarcoma cases that were reported in the 1980s and 1990s and included in the SEER data.

Data from the SEER program indicate that African Americans are clearly more predisposed to develop sarcomas than are whites (with the exception of blood vessel sarcomas, which in this scheme included Kaposi's sarcoma) (Table 1) (5). The predilection for development of sarcomas in males was shown for all histologic subtypes except stromal sarcomas and leiomyosarcomas. The higher incidence of leiomyosarcomas in females is probably due to the occurrence of uterine leiomyosarcoma.

Approximately $40 \%$ of all sarcomas occur in people older than 55 years. The incidence rate in the general population is 1.4 per 100,000 but rises to 8 per 100,000 for people older than 80 years. The distribution of histologic types varies by age. For example, rhabdomyosarcoma is mostly a tumor of the young; synovial sarcoma and fibrosarcoma occur in younger adults; and malignant fibrous histiocytomas occur more often in mature adults (6).

In contrast to the biologic behavior of carcinomas, which varies dramatically depending on the site and therefore the cell type of origin, that of soft tissue sarcomas is similar regardless of
Table 1. Average annual age-adjusted incidence rates (per 100,000) for soft tissue sarcoma by histologic type, race, and gender*

\begin{tabular}{lrrrrc}
\hline \multirow{2}{*}{ Histology } & \multicolumn{2}{c}{ White } & & \multicolumn{2}{c}{ African American } \\
\cline { 2 - 3 } & Men & Women & & Men & Women \\
\hline Blood vessel sarcoma & 6.81 & 0.28 & & 5.04 & 0.32 \\
Fibrosarcoma & 1.54 & 1.09 & & 1.88 & 1.48 \\
Leiomyosarcoma & 0.80 & 1.10 & & 1.22 & 2.01 \\
Sarcoma, NOS & 0.53 & 0.44 & & 0.72 & 0.50 \\
Liposarcoma & 0.60 & 0.35 & & 0.79 & 0.31 \\
Rhabdomyosarcoma & 0.32 & 0.17 & & 0.43 & 0.22 \\
Stromal sarcoma & 0.02 & 0.23 & & 0.03 & 0.34 \\
Synovial sarcoma & 0.10 & 0.09 & & 0.17 & 0.07 \\
Meningiosarcoma & 0.07 & 0.09 & & 0.20 & 0.08 \\
Mesenchymoma & 0.01 & $<0.01$ & & 0.03 & 0.04 \\
Lymphangiosarcoma & $<0.01$ & 0.01 & & - & - \\
Others & 0.35 & 0.67 & & 0.23 & 1.06 \\
Total & 11.15 & 4.53 & & 10.73 & 6.43 \\
\hline
\end{tabular}

*Reprinted from reference 5 .

NOS indicates not otherwise specified.

location. However, the management and therefore the outcome for these lesions are based largely on anatomic location. Thus, a general division of sarcomas into those of the extremities and those of visceral, retroperitoneal, or trunk origin is convenient for discussion. This review focuses primarily on sarcoma of the extremities.

\section{CLASSIFICATION AND GRADING}

Sarcomas are classified according to the type of tissue that they form, not where they arise; thus, a histogenic classification is used. Most connective tissues, if not all, can potentially give rise to either a benign or malignant type of tumor (Table 2). According to data from Memorial Sloan-Kettering Cancer Center, the most common sarcoma found in the extremities is malignant fibrous histiocytoma, followed by liposarcoma, synovial

From the Department of Surgery, Baylor University Medical Center, Dallas, Texas. Presented at surgical grand rounds, Baylor University Medical Center, January 15, 2003.

Corresponding author: Blake A. Morrison, MD, Department of Surgery, Baylor University Medical Center, 3500 Gaston Avenue, Dallas, Texas 75246. 
Table 2. Histogenic classification scheme for benign and malignant soft tissue tumors*

\begin{tabular}{lll}
\hline $\begin{array}{l}\text { Tissue formed } \\
\text { (histogenesis) }\end{array}$ & $\begin{array}{l}\text { Benign } \\
\text { soft tissue tumor }\end{array}$ & $\begin{array}{l}\text { Malignant } \\
\text { soft tissue tumor }\end{array}$ \\
\hline Fat & Lipoma & Liposarcoma \\
Fibrous tissue & Fibroma & Fibrosarcoma \\
Skeletal muscle & Rhabdomyoma & Rhabdomyosarcoma \\
Smooth muscle & Leiomyoma & Leiomyosarcoma \\
Bone & Osteoma & Osteosarcoma \\
Cartilage & Chondroma & Chondrosarcoma \\
Synovium & Synovioma & Synovial sarcoma \\
Blood vessel & Hemangioma & Angiosarcoma \\
Lymphatics & Lymphangioma & Lymphangiosarcoma; \\
& & malignant hemangio- \\
& pericytoma \\
Nerve & Neurofibroma & Neurofibrosarcoma \\
Mesothelium & Benign mesothelioma & Malignant mesothelioma \\
Tissue histiocyte & Benign fibrous & Malignant fibrous \\
& histiocytoma & histiocytoma \\
Pluripotent & None recognized & Malignant mesenchymoma \\
Uncertain & None recognized & Ewing's sarcoma; alveolar \\
& & soft parts sarcoma; \\
& & epithelioid sarcoma \\
\hline
\end{tabular}

*Reprinted with permission from reference 1.

sarcoma, fibrosarcoma, leiomyosarcoma, and then malignant peripheral nerve tumor (7).

In general, although some sarcomas are clearly more aggressive than others, the specific histologic type appears to be of secondary importance in predicting the biologic behavior. This behavior is best predicted by the histologic grade, which is determined by 4 factors: mitotic index, degree of cellularity, necrosis when present, and degree of nuclear anaplasia.

\section{STAGING}

The 2002 American Joint Committee on Cancer staging scheme for sarcomas is shown in Table 3 (1). This staging system is like most tumor-node-metastasis (TNM) systems, but it also includes $\mathrm{G}$ for histologic grade. $\mathrm{T} 1$ tumors are $\leq 5 \mathrm{~cm} ; \mathrm{T} 2,>5 \mathrm{~cm}$. Both $\mathrm{T} 1$ and $\mathrm{T} 2$ tumors are designated $\mathrm{a}$ for superficial and $\mathrm{b}$ for deep. NO indicates no disease-positive lymph nodes, while N1 indicates positive lymph nodes. With sarcoma, a positive lymph node is considered the same as any metastasis. MO indicates no metastases; M1, distant metastases. The histologic grade varies somewhat depending on the institution. Some institutions use a 2 -tiered system, designating low grade or high grade. Some use G1 for well differentiated, G2 for moderately differentiated, G3 for poorly differentiated, and G4 for more poorly differentiated or undifferentiated. Many institutions use a 3-tiered system.

Staging can be summarized as follows:

- Stage I-low-grade lesions without metastasis, regardless of the lesion's size or location

- Stage III-high-grade lesions that are large and deep

- Stage IV-lesions with metastasis (whether in a lymph node or a distant location), regardless of the lesion's size, location, or histologic grade
Table 3. Staging of soft tissue sarcomas of the extremities*

\section{Stage Grouping}

Stage I T1a, 1b, 2a, 2b N0 M0 G1-2 G1 Low

Stage II T1a, 1b, 2a NO M0 G3-4 G2-3 High

$\begin{array}{llllll}\text { Stage III T2b No } & \text { M0 } & \text { G3-4 } & \text { G2-3 } & \text { High }\end{array}$

Stage IV Any T

Any $T$

N1 M0 Any G Any G High or Low

No M1 Any G Any G High or Low

\section{Definition of TNM}

Primary Tumor $(T)$

TX Primary tumor cannot be assessed

TO No evidence of primary tumor

T1 Tumor $5 \mathrm{~cm}$ or less in greatest dimension

T1a superficial tumor

T1b deep tumor

T2 Tumor more than $5 \mathrm{~cm}$ in greatest dimension

T2a superficial tumor

T2b deep tumor

Regional Lymph Nodes (N)

NX Regional lymph nodes cannot be assessed

NO No regional lymph node metastasis

$\mathrm{N} 1$ * Regional lymph node metastasis

*Note: Presence of positive nodes (N1) is considered stage IV.

\section{Distant Metastasis (M)}

MX Distant metastasis cannot be assessed

MO No distant metastasis

M1 Distant metastasis

\section{Histologic Grade (G)}

GX Grade cannot be assessed

G1 Well differentiated

G2 Moderately differentiated

G3 Poorly differentiated

G4 Poorly differentiated or undifferentiated (four-tiered systems only)

*Used with the permission of the American Joint Committee on Cancer (AJCC), Chicago, Illinois. The original source for this material is the AJCC Cancer Staging Manual, Sixth Edition (2002) published by Springer-Verlag New York, www.springer-ny.com.

- Stage II-all other lesions

In the 1997 staging system, T2 lesions that were low grade were classified as stage IIa disease. Those lesions have been reclassified in the 2002 staging system and moved into stage I. This change reflects the observation that patients with large, deep, low-grade lesions have a better outcome than those with stage II lesions.

The staging systems for sarcoma remain controversial. One of the problems is the lack of specific staging for the tumor's location. Extremity, head and neck, visceral, and retroperitoneal sarcomas are all combined, so it is impractical to associate a stage with any kind of uniform surgical approach. Another problem is the failure to include biologic subtypes, some of which are much more aggressive than others. For example, synovial sarcomas and rhabdomyosarcomas are often very aggressive, and yet they may appear to be low-grade or small tumors. Conversely, well-differentiated liposarcomas and myxoid sarcomas have very low metastatic potential irrespective of their size.

The confusion is compounded by the fact that tumor behavior can vary considerably within a histologic subtype. An example is fibrosarcoma. The infantile version, even with a high 
number of mitotic figures, tends to have very low metastatic potential compared with an adult fibrosarcoma of the same grade, which would be a fairly aggressive tumor.

\section{ETIOLOGY}

In 1940, James Ewing, of Ewing's sarcoma fame, wrote: "Of the specific etiology of sarcoma, little is definitely known" (8). This is largely still true today, although some etiologic associations are known for a minority of sarcomas. These associated factors include genetic predisposition, exposure to radiation or chemical carcinogens, immunologic and viral factors, and environmental factors.

An association between trauma and soft tissue sarcoma has been suggested for over 200 years; in almost all instances, the relationship is probably not causal. Patients may report a history of trauma, but instead of causing the mass, the trauma probably directed their attention to it. Rare exceptions include dermatofibrosarcoma arising in a burn scar or fibrosarcomas arising at old orthopaedic fracture sites or in the surgical scars of patients with Gardner's syndrome. Gardner's syndrome is an inherited disorder characterized by clonic polyposis and multiple benign as well as some malignant neoplasms.

A genetic predisposition clearly exists in a minority of these tumors, such as Gardner's syndrome. Retinoblastoma is also associated with osteosarcomas, and this fact has led to the discovery that many sarcomas display structural defects of the RB1 gene. Multiple endocrine neoplasia 2B syndrome includes ganglioneuromas, and patients with neurofibromatosis, or von Recklinghausen's disease, carry a $7 \%$ to $10 \%$ lifetime risk of developing malignant neurofibrosarcoma. Li-Fraumeni cancer family syndrome includes an increased susceptibility to a variety of sarcomas. Many patients with Li-Fraumeni syndrome have a defect in the p53 gene, and this tumor suppressor gene is defective in some types of sarcoma as well (9).

Ionizing radiation is associated with sarcoma and acts as both a tumor initiator and a promoter. Of the radiation-induced sarcomas, approximately $70 \%$ are malignant fibrous histiocytoma, which tends to be an aggressive type of tumor. The latency period is 7 to 25 years, and these patients typically have a poor prognosis. In one series of 53 patients, a 5-year survival rate of 26\% was reported (10).

Chemical carcinogens have been reported to be etiologic agents. Although the effects of these carcinogens are much better described in experimental animals, the best human example is probably the dioxin-containing herbicide TDCC, or Agent Orange, a defoliant that was widely used in the Vietnam War. Agent Orange was implicated to such an extent that the Department of Veterans Affairs has agreed to compensate veterans who were exposed to it and developed sarcomas. Follow-up studies on the subject have yielded mixed results. Collins and coworkers found no causal relationship between dioxin exposure in factory workers and the incidence of sarcoma (11). However, Fingerhut et al (12) and Suruda et al (13) demonstrated a significant increase in risk of death from sarcoma among exposed factory workers.

Immunologic factors are implicated as well. Immunosuppression is associated with an increased risk of all kinds of neoplasms, typically those that are epithelial in origin (e.g., squamous cell carcinoma of the skin) and lymphomas. Sarcomas, however, also have been described.

Viral factors are clearly at play. In 1911, Peyton Rous demonstrated a transmissible avian sarcoma virus, named the Rous virus, and later won the Nobel Prize for this discovery. HIV is probably the best-known example in humans, being implicated in Kaposi's sarcoma. It is unclear, however, whether Kaposi's sarcoma is caused by the virus alone or by a combination of viral effect and immunologic suppression (probably the latter).

Other environmental factors are also to blame. The link between mesothelioma and asbestos is well known, as is the link between Stewart-Treves syndrome (postmastectomy lymphangiosarcoma) and long-standing lymphedema of the extremities. Interestingly, a risk of lymphangiosarcoma exists after mastectomy and radiation therapy even when the upper extremity appears grossly normal, and the tumor may not be in the irradiated field.

The link between foreign bodies and sarcoma has been described since the 1880s and has been well studied in rats. Rare human cases have also been described, involving surgical implants, plastic, bullets, surgical sponges, bone wax, or TeflonDacron prostheses. The largest study was done by Engel and colleagues, who looked at the rate of sarcoma in thousands of women with breast implants but did not find a statistically significant increase (14).

\section{DIAGNOSIS}

The majority of patients with sarcoma present with a painless mass, although as many as 33\% complain of pain, which may indicate a poor prognosis (7). Of the patients who present with pain, about half eventually undergo amputation; rapidly growing or aggressive tumor subtypes are more likely to be painful. Pain is often the first complaint in patients with malignant peripheral nerve tumors, which tend to spread along nerve bundles and are difficult to control.

Unfortunately, a prolonged delay in diagnosis is common. As many as half of patients wait for months to seek treatment for the mass, and the treating physician may entertain a diagnosis of a pulled muscle or traumatic hematoma for some time. These diagnoses should be considered only when there is a clear-cut history of trauma, and the physician should set a time limit of 6 to 8 weeks for observing the mass before treating it.

When evaluating a patient who has a potentially malignant mass, the physician should obtain these important elements of the history: 1) the duration of symptoms; 2) any recent change in size or consistency of the mass; 3 ) the constellation of symptoms, including pain, fever, or paresthesias; and 4) an antecedent history of trauma or other environmental factors, such as irradiation to the field, history of mastectomy, etc.

Six factors to assess in the physical examination include the location of the mass; its shape, size, consistency, and relation to surrounding tissues; and the state of the regional nodes. The nodes should be examined for metastasis even though the spread of sarcoma is almost always hematogenous.

In general, a biopsy should be performed on any soft tissue mass that is symptomatic or enlarging, any new mass that persists $>4$ weeks, or any soft tissue mass that is $>5 \mathrm{~cm}$ in diameter.

The method of biopsy is critical and will vary somewhat, depending on the institution and the pathologist's experience. 
Traditionally, closed biopsy techniques such as fine-needle aspiration (FNA) or core-needle biopsy (CNB) have had limited roles in diagnosing suspected sarcomas because of small sample size and questionable accuracy. The latter is particularly worrisome because of the highly pleomorphic nature of some sarcomas, which have a widely variable degree of malignancy throughout the mass. However, CNB and FNA are used routinely at some institutions, including M. D. Anderson Cancer Center and Memorial Sloan-Kettering Cancer Center, where a 1997 study reported a $93 \%$ adequacy rate and a $95 \%$ accuracy rate in determining malignancy (15). CNB is used by many surgeons at Baylor University Medical Center as well. Reliable use of this technique requires confidence on the part of the reviewing pathologist as well as a firm understanding of its limitations.

When open biopsy is preferred by the surgeon or pathologist, or if CNB/FNA returns an inadequate specimen, then excisional or incisional biopsy is required. For a small mass, 3 to $5 \mathrm{~cm}$ in its largest dimension, an excisional biopsy is appropriate. An incisional biopsy should be performed on masses $>5 \mathrm{~cm}$. Impeccable technique is critical: the surgeon must plan the biopsy with the intention of coming back for a major resection if the worstcase scenario, sarcoma, proves to be the diagnosis. The biopsy incision must be oriented along the long axis of the extremity, and a minimal amount of skin flap should be raised superficial to the incision. Meticulous hemostasis is essential to prevent the inadvertent dissemination of tumor cells.

In general, a diagnosis from frozen sections should be relied upon with extreme caution. Definitive treatment of a malignant lesion should be deferred until the results from the permanent sections and the results of an appropriate metastatic workup, including a computed tomography (CT) scan of the chest, have been reviewed. The lung is the most common site of metastasis for sarcomas, so a chest CT scan is typically indicated for all patients with newly diagnosed sarcoma. Other sites of metastasis vary by the type of lesion. An example would be angiosarcoma, which metastasizes to bone and thus may be an indication for a bone scan.

A preoperative imaging study is indicated in all cases of sarcoma, and both CT and magnetic resonance imaging (MRI) are used. Plain films are of limited overall value. In general, MRI is considered superior for studying soft tissues, but CT is widely used as well. The choice of imaging method is a function of the surgeon's preference and the experience of the institution's radiology staff.

\section{SURGICAL TREATMENT}

Surgery is the mainstay of treatment for soft tissue sarcomas, and surgical interventions have spanned the spectrum from simple wide local excision to radical amputation. Early attempts at limited resection of these tumors resulted in uniformly poor results because of local recurrence, so radical amputation became the accepted surgical treatment.

In the past 2 decades, however, the pendulum has swung back in favor of limb-salvage techniques. With multimodal approaches, limb-sparing resections can be achieved, depending on the institution, in as many as $90 \%$ of patients, with an overall local recurrence rate of $<10 \%$ (1). Compartment excision, one of the earliest limb-sparing techniques, was developed because of the potential for various tumors to spread microscopically along the muscle groups. The goal is to remove that group or groups of muscles from origin to insertion. Although simple wide local resection with adjuvant therapy has become the mainstay of limb-salvage techniques, there may still be a place for compartment excision. An example would be the presence of a small but otherwise aggressive tumor type located within a muscle group whose excision can afford a cure, such as the adductor of the thigh or the quadriceps.

Compartment excision requires that the surgeon identify 3 things before marking the incisions: 1 ) the location of the old biopsy incision, 2) the presumed extent of the tumor, and 3) where skin flaps should be. As with the biopsy, the surgeon should carefully avoid touching the tumor itself, try to manipulate tissues around the tumor without ever exposing the tumor, and keep a $3-\mathrm{cm}$ margin if possible. When this is not feasible due to the deep margin, the surgeon should try to always get at least one uninvolved fascial plane.

For a wide local excision, the incisions are based on the extent of the tumor plus at least a $3-\mathrm{cm}$ margin, without any particular anatomic landmarks. Again, the surgeon must pay meticulous attention to hemostasis, try not to touch the tumor, and get 3- to 5-cm margins all the way around. If necessary, involved major vascular structures, which should have been revealed by the preoperative imaging studies, can be resected and then replaced with interposition grafts. The previous biopsy site should be included in any resection. This includes the previous scar for incisional or excisional biopsies and the puncture site and entire needle tract for FNA or CNB.

Contraindications to limb-sparing techniques include instances in which an underlying defect persists through the entire extremity, as in the Stewart-Treves syndrome. In the case of lymphangioma arising from lymphedema, it makes no sense to simply resect the tumor and leave other at-risk tissue behind. The same concept holds true for malignant schwannomas in a patient with neurofibromatosis. These patients are candidates for amputation. Recurrence alone, however, is not an indication for amputation when these patients can undergo additional wide local excision.

Amputation is also indicated when resection will not leave an anatomically functional limb. Some proximal extremity lesions require amputation. A lesion in the very proximal arm may require forequarter amputation; likewise, a lesion in the proximal thigh or groin may require hemipelvectomy or hip disarticulation. More distal lesions can be cured with limited amputation, such as a transmetatarsal amputation for a lesion in the toe or a digital ray amputation for a lesion in a finger or hand.

\section{ADJUVANT THERAPY}

Adjuvant therapy is important even though surgical excision is the mainstay of treatment. For small, low-grade tumors, wide local excision alone provides very good results. Most patients, however, will go on to receive either external-beam radiation therapy (EBRT) or perioperative brachytherapy from an implanted source. Patients with low-grade lesions $>5 \mathrm{~cm}$ in diameter are typically offered EBRT. Patients with high-grade lesions between 5 and $10 \mathrm{~cm}$ in diameter can be given either EBRT or brachytherapy. Patients with very large $(\geq 10 \mathrm{~cm})$, high-grade le- 


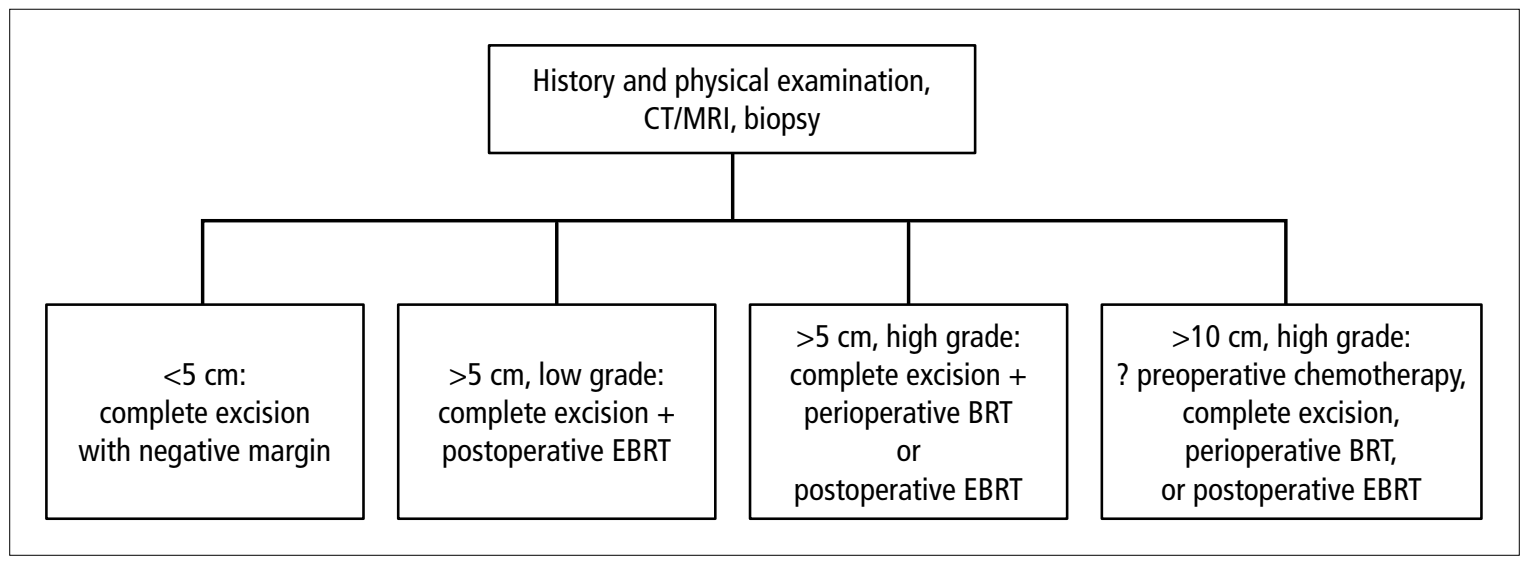

Figure. Algorithm for diagnosis and treatment of soft tissue sarcoma of the extremities developed at Memorial Sloan-Kettering Cancer Center. Reprinted with permission from reference 7. CT indicates computed tomography; MRI, magnetic resonance imaging; EBRT, external-beam radiation therapy; BRT, brachytherapy.

Table 4. Five-year survival rates in extremity soft tissue sarcoma*

\begin{tabular}{ccccc}
\hline Stage & N & $\begin{array}{c}\text { Freedom from } \\
\text { local recurrence }\end{array}$ & $\begin{array}{c}\text { Disease-free } \\
\text { survival }\end{array}$ & $\begin{array}{c}\text { Overall } \\
\text { survival }\end{array}$ \\
\hline I & 137 & $88.04 \%$ & $86.13 \%$ & $90.00 \%$ \\
II & 491 & $81.97 \%$ & $71.68 \%$ & $80.89 \%$ \\
III & 469 & $83.44 \%$ & $51.77 \%$ & $56.29 \%$
\end{tabular}

* Local recurrence, disease-free survival, and overall survival by stage. Source: Memorial Sloan-Kettering Cancer Center for the time period of 7/1/82 to 6/30/00. Used with the permission of the American Joint Committee on Cancer (AJCC), Chicago, Illinois. The original source for this material is the AJCC Cancer Staging Manual, Sixth Edition (2002) published by Springer-Verlag New York, www.springer-ny.com.

sions are considered for neoadjuvant therapy with chemoradiation - a radiation sensitizer and either EBRT or brachytherapy. The radiation sensitizer is usually doxorubicin or ifosfamide based.

Adjuvant therapy using doxorubicin- or ifosfamide-based regimens is still somewhat controversial, largely because the survival benefit has not been uniform from center to center (1). Exceptions include rhabdomyosarcoma and Ewing's sarcoma, which are very sensitive to and should be treated with adjuvant chemotherapy. Isolated limb perfusion therapy using cytotoxic agents such as melphalan, tumor necrosis factor, and interferons holds great promise but remains investigational (16).

The Figure illustrates a treatment algorithm proposed by the Memorial Sloan-Kettering Cancer Center group, starting with history, physical examination, preoperative imaging study (CT or MRI), and biopsy (7). Small lesions are treated with complete excision, as long as a negative margin can be obtained. Depending on their size and histologic grade, larger lesions are treated with various combinations of complete excision and pre- or postoperative radiation therapy and, for very large lesions, neoadjuvant chemoradiation.

\section{OUTCOME AND FOLLOW-UP}

Table 4 shows outcome data from Memorial Sloan-Kettering Cancer Center from the period 1982 to 2000. Reasonably good results were reported for stage I tumors, with a disease-free survival rate of $86 \%$ and freedom-from-recurrence rate of $88 \%$. The rates for stage III lesions go down to about $52 \%$ and $83 \%$, respec- tively. Data for stage IV lesions are not shown, probably because they are uniformly dismal.

Regular follow-up is very important for these patients, at least annually if not more frequently for the first 5 years. The followup for high-grade lesions should be directed at both local recurrence and metastatic disease, whereas local recurrence is the primary concern for low-grade lesions. Follow-up must be continued for the long term, as low-grade lesions can recur as late as 20 years after the original resection. In fact, patients who are clear of disease 5 years after resection still have a 10\% risk of local recurrence (17).

Local recurrence is not a death sentence in extremity sarcoma as it often is in retroperitoneal sarcoma. These patients can still be treated with limb-salvage methods when technically feasible, and the survival rate approaches that of previously untreated patients. Pulmonary metastases - even multiple or bilateral metastases-are typically resected, and aggressive resection is associated with a cure in $25 \%$ to $35 \%$ of patients (1).

Patients with unresectable metastases or extrapulmonary metastases are offered adjuvant chemotherapy. Occasionally, patients with bony or cerebral metastases are offered palliative EBRT.

\section{CONCLUSION}

Soft tissue sarcomas of the extremities are rare and challenging neoplasms, and every general surgeon is likely to face one at least once or twice in his or her career. Recurrence of extremity sarcoma is not a death sentence, and these patients should be treated aggressively. When providing definitive therapy, the general surgeon should involve colleagues from appropriate related services (e.g., orthopaedics, vascular surgery, plastic surgery, oncology, radiation therapy) early in the process. Prompt diagnosis and the appropriate choice of biopsy method are vital in treating a potentially malignant soft tissue mass.

1. Sondak VK. Sarcomas of bone and soft tissue. In Greenfield LJ, Mulholland MW, Oldham KT, Zelenock GB, Lillemoe KD, eds. Surgery: Scientific Principles and Practice, 3rd ed. Philadelphia: Lippincott Williams \& Wilkins, 2000:2258-2280.

2. Parker SL, Tong T, Bolden S, Wingo PA. Cancer statistics, 1996. CA Cancer J Clin 1996;46:5-27. 
3. Das Gupta TK, Chaudhuri PK. Characteristics of soft tissue sarcomas. In Das Gupta TK, Chaudhuri PK, eds. Tumors of the Soft Tissues, 2nd ed. Stanford: Appleton and Lange, 1998:3-9.

4. Devesa SS, Blot WJ, Stone BJ, Miller BA, Tarone RE, Fraumeni JF Jr. Recent cancer trends in the United States. J Natl Cancer Inst 1995;87:175-182.

5. Zahm SH, Fraumeni JF Jr. The epidemiology of soft tissue sarcoma. Semin Oncol 1997;24:504-514.

6. Enzinger FM, Weiss SW. Soft Tissue Tumors, 2nd ed. St. Louis: CV Mosby, 1988:2.

7. Brennan MF, Lewis JJ. Soft tissue sarcomas and bone tumors. In Townsend CM, Beauchamp DR, Evers MB, Mattox KL, eds. Sabiston Textbook of Surgery: The Biological Basis of Modern Surgical Practice, 16th ed. Philadelphia: WB Saunders Co, 2001:511-517.

8. Ewing J. Neoplastic Diseases: A Treatise on Tumors, 4th ed. Philadelphia: WB Saunders, 1940:274.

9. Raaf JH. Soft Tissue Sarcomas: Diagnosis and Treatment. St. Louis: MosbyYear Book Inc, 1993:4.

10. Laskin WB, Silverman TA, Enzinger FM. Postradiation soft tissue sarcomas. An analysis of 53 cases. Cancer 1988;62:2330-2340.

11. Collins JJ, Strauss ME, Levinskas GJ, Conner PR. The mortality experience of workers exposed to 2,3,7,8-tetrachlorodibenzo-p-dioxin in a trichlorophenol process accident. Epidemiology 1993;4:7-13.
12. Fingerhut MA, Halperin WE, Marlow DA, Piacitelli LA, Honchar PA, Sweeney MH, Greife AL, Dill PA, Steenland K, Suruda AJ. Cancer mortality in workers exposed to 2,3,7,8-tetrachlorodibenzo-p-dioxin. N Engl J Med 1991;324:212-218.

13. Suruda AJ, Ward EM, Fingerhut MA. Identification of soft tissue sarcoma deaths in cohorts exposed to dioxin and to chlorinated naphthalenes. Epidemiology 1993;4:14-19.

14. Engel A, Lamm SH, Lai SH. Human breast sarcoma and human breast implantation: a time trend analysis based on SEER data (1973-1990). J Clin Epidemiol 1995;48:539-544.

15. Heslin MJ, Lewis JJ, Woodruff JM, Brennan MF. Core needle biopsy for diagnosis of extremity soft tissue sarcoma. Ann Surg Oncol 1997;4:425-431.

16. Plaat BE, Molenaar WM, Mastik MF, Koudstaal J, van den Berg E, Koops HS, Hoekstra HJ. Hyperthermic isolated limb perfusion with tumor necrosis factor-alpha and melphalan in patients with locally advanced soft tissue sarcomas: treatment response and clinical outcome related to changes in proliferation and apoptosis. Clin Cancer Res 1999;5:1650-1657.

17. Lewis JJ, Leung D, Casper ES, Woodruff J, Hajdu SI, Brennan MF. Multifactorial analysis of long-term follow-up (more than 5 years) of primary extremity sarcoma. Arch Surg 1999;134:190-194. 